\title{
Le tocilizumab pour les patients hospitalisés atteints de la COVID-19
}

\author{
Kevin Afra MD MASS, Luke Y.C. Chen MD MMÉd, David Sweet MD
}

Citation : CMAJ 2021 April 12;193:E521. doi : 10.1503/cmaj.210066-f; diffusion hâtive le 10 mars 2021

Voir la version anglaise de l'article ici : www.cmaj.ca/lookup/doi/10.1503/cmaj.210066

\section{1} Le tocilizumab est un médicament immunomodulateur qui bloque l'activité de l'interleukine-6 (IL-6)

De nombreux patients atteints de la forme grave de la maladie à coronavirus 2019 (COVID-19) présentent des ratés immunitaires caractérisés par une réponse défaillante de l'interféron, suivie par une élévation des cytokines pro-inflammatoires, IL- 6 notamment $^{1}$. Le tocilizumab est un anticorps monoclonal du récepteur IL-6 dont l'utilisation est homologuée pour les affections inflammatoires².

Le tocilizumab diminue le taux de mortalité chez les patients atteints des formes graves à critiques de la COVID-19

On a réalisé 2 vastes essais cliniques randomisés et contrôlés évaluant l'effet du tocilizumab chez des patients hospitalisés atteints de la COVID-19. L'essai REMAP-CAP (Randomized, Embedded, Multifactorial Adaptive Platform Trial for Community-Acquired Pneumonia) portant sur des patients dont l'état de santé était critique, a révélé que le tocilizumab était supérieur à la norme thérapeutique pour le taux de mortalité à 21 jours et le nombre de jours sans soutien des fonctions vitales ${ }^{3}$. Le taux de mortalité perhospitalière était de $28,0 \%$ pour le groupe traité au tocilizumab contre $35,8 \%$ pour le groupe traité selon la norme thérapeutique (nombre de sujets à traiter [NST] = 12). La prépublication de l'essai RECOVERY (Randomised Evaluation of COVID-19 Therapy) a démontré que le tocilizumab améliore le taux de mortalité à 28 jours : $29,5 \%$ des patients du groupe traité au tocilizumab sont décédés contre $33,1 \%$ des patients traités selon la norme thérapeutique $(\mathrm{NST}=27)^{4}$. Cet avantage s'additionne à celui offert par les corticostéroïdes.

Les patients hospitalisés atteints de la COVID-19 dont l'état de santé n'est pas critique profitent aussi de l'utilisation du tocilizumab

Les patients participant à l'essai RECOVERY étaient admissibles au traitement par le tocilizumab s'ils présentaient de l'hypoxie (saturation en oxygène $<92 \%$ à l'air ambiant ou nécessitant une oxygénothérapie) ou une réponse inflammatoire systémique (protéine $C$ réactive $\geq 75 \mathrm{mg} / \mathrm{L}$ ). Dans le cadre de cet essai, les avantages du tocilizumab semblaient cohérents pour tous les niveaux de soutien respiratoire initiaux $x^{4}$.
4 . On devrait administrer du tocilizumab de façon précoce aux patients admissibles On ne devrait pas retarder les traitements des patients qui sont admissibles à l'utilisation du tocilizumab. Les seuls essais qui ont démontré un avantage sur le taux de mortalité, l'essai RECOVERY et l'essai REMAP-CAP, ont administré du tocilizumab dans les phases initiales de l'hospitalisation (durée médiane : 1-2 jours) 3,4.

\section{L'innocuité du tocilizumab semble démontrée pour le traitement de la COVID-19}

L'hypersensibilité, la cytopénie, des lésions hépatiques et des perforations gastrointestinales sont des effets indésirables importants du tocilizumab, mais qui ont rarement été observés dans les essais en lien avec la COVID-19. Par contre, on a exclu de l'essai REMAP-CAP les patients présentant un niveau d'aminotransférase 5 fois plus grand que la limite supérieure normale ou une numération plaquettaire inférieure à $50 \times 10^{9} / \mathrm{L}^{3}$.

\section{Références}

1. Chen LYC, Hoiland RL, Stukas S, et al. Confronting the controversy: interleukin- 6 and the COVID-19 cytokine storm syndrome. Eur Respir J 2020;56:2003006.

2. Product monograph: PrActemra (tocilizumab). Mississauga (ON): Hoffmann-La Roche; 2010 Apr. 30, revised 2021 Jan. 4. Accessible ici : https://pdf.hres.ca/dpd_pm/00059560.PDF (consulté le 12 fév. 2021).

3. REMAP-CAP Investigators; Gordon AC, Mouncey PR, Al-Beidh $F$, et al. Interleukin-6 receptor antagonists in critically ill patients with COVID-19. N Engl J Med 2021 Feb. 25 [cyberpublication avant impression]. doi: 10.1056/NEJMoa2100433.

4. RECOVERY Collaborative Group; Horby PW, Pessoa-Amorim G, Peto L, et al. Tocilizumab in patients admitted to hospital with COVID-19 (RECOVERY): preliminary results of a randomised, controlled, open-label, platform trial [pré-impression]. medRxiv 2021 Feb. 11. doi: 10.1101/2021.02.11.21249258. 
Intérêts concurrents : Kevin Afra et Luke Chen sont membres et David Sweet est président du Comité thérapeutique de la ColombieBritannique contre la COVID-19 (B.C. COVID-19 Therapeutics Committee), qui offre des lignes directrices fondées sur les travaux de recherche les plus récents portant sur l'utilisation de thérapies dans la prise en charge de la COVID-19. Luke Chen a obtenu le soutien de GlaxoSmithKline pour un panneau publicitaire portant sur le mepolizumab. Aucun autre intérêt concurrent n'a été déclaré.

Cet article a été révisé par des pairs.

Affiliations : Division des maladies infectieuses (Afra) et Division d'hématologie (Chen), Département de médecine; Département de médecine d'urgence (Sweet); et Centre for Health Education Scholarship (Chen), Université de la Colombie-Britannique; Division de médecine des soins intensifs (Sweet), Service de médecine, Hôpital général de Vancouver, C.-B.; Division des maladies infectieuses (Afra), Département de médecine, Autorité sanitaire Fraser, Surrey, C.-B.

Propriété intellectuelle du contenu : Il s'agit d'un article en libre accès distribué conformément aux modalités de la licence Creative Commons Attribution (CC BY-NC-ND 4.0), qui permet l'utilisation, la diffusion et la reproduction dans tout médium à la condition que la publication originale soit adéquatement citée, que l'utilisation se fasse à des fins non commerciales (c.-à-d., recherche ou éducation) et qu'aucune modification ni adaptation n'y soit apportée. Voir : https://creativecommons.org/licenses/by-nc-nd/4.0/deed.fr.

Remerciements : Les auteurs voudraient reconnaître et remercier le dévouement et le dur labeur du Comité thérapeutique de la ColombieBritannique contre la COVID-19 tout au long de la pandémie.

Correspondance : Kevin Afra, kevin.afra@fraserhealth.ca 